Relations industrielles

Industrial Relations

\title{
Ces entreprises qui font la Chine. Par Dominique Jolly, Paris : Eyrolles/Editions d'organisation, collection Stratégie, 2011, 255 p., ISBN : 978-2-212-55231-7.
}

\section{Diane-Gabrielle Tremblay}

Volume 67, numéro 3, 2012

URI : https://id.erudit.org/iderudit/1012546ar

DOI : https://doi.org/10.7202/1012546ar

Aller au sommaire du numéro

Éditeur(s)

Département des relations industrielles de l’Université Laval

ISSN

0034-379X (imprimé)

1703-8138 (numérique)

Découvrir la revue

Citer ce compte rendu

Tremblay, D.-G. (2012). Compte rendu de [Ces entreprises qui font la Chine. Par Dominique Jolly, Paris : Eyrolles/Éditions d'organisation, collection Stratégie, 2011, 255 p., ISBN : 978-2-212-55231-7.] Relations industrielles / Industrial Relations, 67(3), 546-548. https://doi.org/10.7202/1012546ar

Tous droits réservés (C Département des relations industrielles de l’Université Laval, 2012
Ce document est protégé par la loi sur le droit d'auteur. L’utilisation des services d'Érudit (y compris la reproduction) est assujettie à sa politique d'utilisation que vous pouvez consulter en ligne.

https://apropos.erudit.org/fr/usagers/politique-dutilisation/ 
mined to fight the trusteeships, are devoted to combating allies rather than organizing new workers. Such is the consequence of a labour organization that, in the words of an SEIU critic cited in Civil Wars, wants to be "the Wal-Mart of unions" (p. 221). Moving towards a corporate and efficiencybased union structure does not stop with trusteeships. Early provides an examination of the SEIU's deployment of call centres as a mechanism through which the union interfaces with members. The project was sold as a comeback strategy, but it is arguable that such a system is required to manage the SEIU's centralized administration of over two million members.

In both books Early asks what has come from Stern's non-adversarial approach with business and his mission to abandon the trade union movement's supposed "class struggle mentality" (Civil Wars, p. 61). Dissident SEIU members and critics referenced in Civil Wars claim that the union's leadership has cut deals with companies behind members' backs, to the detriment of their benefits and pay. That said, in 2005, the SEIU won a hard-fought victory for child care workers represented by Local 880 in Illinois, through a mix of direct action tactics and political lobbying.

Early remains skeptical that the SEIU has made real gains for some of the poorest workers, despite concessions and Stern's appeal to "responsible unions" (Civil Wars, p. 236). As Sal Rosselli, former president of the embattled UHW comments, "Stern's multimillion dollar fights have diverted resources away from healthcare reform and employee free choice, weakening the former and scuttling the latter" (Civil Wars, p. 284). Rosselli is speaking to organized labour's response to Obamacare and the virtual abandonment of the Employee Free Choice Act - one of President Obama's campaign commitments to the working class and American unions. Stern also left the SEIU unable to pay its bills, undemocratic, and unwilling to defend its members, according to Early.
There are several considerations that the reader is left to contend with. First, Early questions the contributions made by the "60's radicals", like Stern, who took charge of the country's most influential labour unions. It is not an accusation that their leadership has been ineffective, but that the entrenched bureaucratic power of a professional cadre of appointees has been reconstituted with new faces. Early concludes that since the 1990s, there "seemed to be a glib assumption that any solid sixties political resume guaranteed laudatory results in subsequent labor work" (Civil Wars, p. 17). Such assumptions were fanned by labour scholars of the same generation, who saw their peers taking over the offices once occupied by conservative labour leaders. To this point Early is unequivocal: union revitalization is nothing without strong union democracy, and neither academics nor appointed activists can substitute for rank-and-file democratic decision-making. Embedded and Civil Wars are informative, provocative, and important. Even though both speak to U.S. labour history, for Canadian readers there is inspiration in Early's embedded approach to researching and writing about trade unions and the labour movement.

\section{Andrew Stevens}

Queen's University

\section{Ces entreprises qui font la Chine}

Par Dominique Jolly, Paris : Eyrolles/Éditions d'organisation, collection Stratégie, 2011, 255 p., ISBN : 978-2-212-55231-7.

Voici un excellent ouvrage, fort pertinent non seulement pour les gestionnaires ou cadres qui iront en Chine pour affaires, mais aussi pour les étudiants et chercheurs qui s'intéressent à l'évolution récente de ce très grand pays, dominant sur la scène mondiale actuelle. Comme le souligne d'ailleurs I'ancien directeur de Schneider Electric Chine en préface, la Chine attire tous les regards aujourd'hui : "le jeune cadre comme un passage obligé pour sa carrière, l'étudiant 
qui sait que l'avenir se joue en partie en Asie », et on pourrait ajouter les chercheurs travaillant sur la mondialisation et le développement international.

L'ouvrage est très bien documenté, à partir de sources multiples, et surtout, très bien présenté et illustré avec des schémas, des encadrés qui présentent des cas particuliers d'entreprises, ainsi qu'un bon nombre de graphiques et de tableaux statistiques. La première partie de l'ouvrage est particulièrement utile pour les personnes qui veulent en connaître plus sur la dimension politique des relations avec la Chine ou de la mondialisation, puisqu'elle traite de "l'économie socialiste de marché au centre du monde ». Le premier chapitre s'ouvre sur le titre de "China Corporation : une nouvelle légitimité pour l'appareil d'État ». Ce chapitre s'adresse autant au grand public qu'aux chercheurs dans le domaine, puisqu'il fait le point sur l'évolution du rôle du Parti communiste chinois, mais aussi sur la manière dont la Chine a pu promouvoir ses grandes entreprises à l'échelle internationale. L'importance des investissements étrangers est aussi mise en évidence et ici encore, avec nombre d'exemples d'entreprises entrées en bourse. Le chapitre 2 traite des performances économiques innombrables que ce pays a réalisées au cours des dernières décennies. Les données sur le PIB, la balance commerciale, les réserves de change, l'envolée des bourses permettent de bien comprendre la situation économique de la Chine. En fin de chapitre, il y a une synthèse en quelques points, ce qui constitue un aspect très utile pour les lecteurs, et aussi pour l'utilisation pédagogique de l'ouvrage. On trouve également une liste de références bibliographiques à la fin de chaque chapitre, pour ceux qui veulent approfondir la question.

La partie 2 porte sur les stratégies des entreprises étrangères en Chine. On y traite des modes de développement des entreprises et on y présente notamment un tableau sur les divers modes d'entrée des entreprises étrangères en Chine, combinant les ressources internes et externes, ainsi que des stratégies de coentreprise, acquisition, filiale ou bureau de représentation. Une analyse des divers secteurs d'activité permet de mieux comprendre comment la grande distribution comme les secteurs du luxe se sont développés en Chine. L'analyse des secteurs du luxe est particulièrement intéressante et la montée des entreprises du luxe est tout à fait étonnante. Le secteur automobile fait l'objet d'un chapitre en soi (chapitre 5) et illustre les stratégies diversifiées des constructeurs locaux comme des constructeurs étrangers. Un tableau sur les réseaux d'alliances dans le secteur automobile illustre la très grande multiplicité des alliances qui se sont formées sur le territoire chinois. Le chapitre 6 porte finalement sur la mise en œuvre des stratégies pour faire face aux défis de l'insertion locale, du transfert de connaissances, de la gestion des ressources humaines et de l'adaptation des méthodes de gestion en général.

L'auteur conclut sur les transformations et les défis sociétaux de la Chine d'aujourd'hui, mais on retient surtout les défis sur le plan de la gestion des ressources humaines et de l'organisation du travail : une somme de différences à gérer, des échelles de temps différentes, une capacité d'innovation retenue, l'appel du confucianisme à l'harmonie, un rapport à l'autre plus réservé et moins direct qu'en Occident, l'importance de la hiérarchie, la mise de l'avant du groupe ou du collectif, bien que l'auteur précise que le Chinois peut être très collectiviste dans sa famille et son réseau, mais individualiste dans son milieu de travail. Un autre des défis majeurs a trait aux diverses générations présentes sur le marché du travail. L'ancienne génération a surtout travaillé dans des entreprises d'État et n'est pas habituée à évoluer dans un système basé sur la performance. La génération intermédiaire a une meilleure connaissance du marché, de meilleures habiletés linguistiques et de meilleures compétences, mais 
cette génération a été peu exposée à l'international et peut avoir des difficultés d'adaptation culturelle dans le nouveau contexte d'internationalisation de la Chine. Enfin, la nouvelle génération se compose d'une combinaison de locaux, d'émigrés revenus au pays ou d'expatriés. Ceux-ci ont eu une plus grande exposition à l'international, sont plus adaptables et connaissent mieux les langues étrangères, l'anglais principalement. Ces différentes générations se traduisent par des défis différents dans les organisations.

Pour conclure, l'ouvrage est donc une excellente synthèse sur le monde des affaires, l'économie, les secteurs d'activité (luxe, automobile, etc.) et les travailleurs en Chine. Outre le contenu fort instructif, il faut souligner l'excellente présentation graphique de l'ouvrage, le nombre important de tableaux et de schémas fort pertinents, ainsi que la section finale de chaque chapitre, qui en rassemble les " points clés ». Cela en fait donc un ouvrage tout à fait pertinent pour les chercheurs et les étudiants en science politique, en gestion, en relations industrielles et en gestion des ressources humaines.

\section{Diane-Gabrielle Tremblay}

Télé-université, UQAM

\section{Gestion stratégique \\ des ressources humaines}

Par Jean-Yves Le Louarn, Paris : Éditions Liaisons, 2010, 275 p., ISBN : 978-2-87880816-2.

L'ouvrage de Jean-Yves Le Louarn, intitulé Gestion stratégique des ressources humaines, fait partie des contributions francophones attendues dans la mer des écrits disponibles dans la langue de Shakespeare. Après avoir brièvement examiné le contenu de chacun des chapitres composant ce livre, nous présenterons notre appréciation générale de même que quelques pistes à explorer par l'auteur en vue d'une prochaine édition.

La contribution de Le Louarn se compose de onze chapitres d'une longueur et d'une profondeur inégales. Dans le premier chapitre, intitulé « Apprendre la gestion des ressources humaines? », l'auteur prend soin de relever que la gestion des ressources humaines (GRH) fait partie des domaines de la vie des entreprises où la formation n'est toujours pas un prérequis à la pratique et où la recherche scientifique date d'au plus une cinquantaine d'années. Après avoir relevé que la GRH est beaucoup plus qu'une question de bon sens, de flair et d'intuition, Le Louarn présente sa dimension éthique et en vient à conclure que si elle est de plus en plus reconnue comme un ingrédient essentiel du succès des organisations, il importe plus que jamais de l'étudier avec soin car le flou et la complexité y sont partout présents et cela, à fortes doses. Pour y progresser, il faut savoir mettre ses préjugés en veilleuse, tolérer l'ambiguiité et faire preuve d'humilité.

Faisant suite au deuxième chapitre qui se propose d'explorer quelques définitions, composantes et acteurs de la GSRH, le troisième nous amène à comprendre toute l'importance de la notion de chaîne de valeur RH et du rôle central qu'y occupe la théorie du capital humain. Ces repères, qui constituent indéniablement le cœur théorique de cet ouvrage, sont par la suite repris sous diverses formes dans chacun des chapitres suivants.

Après un quatrième chapitre dédié à la présentation détaillée d'un exemple complet de stratégie $\mathrm{RH}$ au sein d'une importante compagnie aérienne américaine, le cinquième tâche d'expliquer au lecteur comment se construit, au quotidien, une telle stratégie $\mathrm{RH}$. À l'aide de quelques exemples réels, Le Louarn démontre bien que ce n'est qu'après avoir défini les principaux enjeux stratégiques de l'organisation que les acteurs peuvent passer à l'alignement des pratiques $\mathrm{RH}$.

Le sixième chapitre porte quant à lui sur les stratégies $\mathrm{RH}$ génériques, les enjeux stratégiques $\mathrm{RH}$ et les objectifs stratégiques $\mathrm{RH}$. Si l'auteur y développe des idées d'une 